\title{
Nutritional Neuroscience
}

\section{Omega-3 PUFAs and vitamin D co-supplementation as a safe-effective therapeutic approach for core symptoms of autism spectrum disorder: case report and literature review \\ --Manuscript Draft--}

\begin{tabular}{|c|c|}
\hline \multicolumn{2}{|l|}{ Manuscript Number: } \\
\hline Article Type: & Case Study \\
\hline Keywords: & $\begin{array}{l}\text { Autism; ASD; omega-3 PUFAs; omega-6 PUFAs; AA/EPA ratio; vitamin D } \\
\text { deficiency; omega-3 supplementation; vitamin D supplementation }\end{array}$ \\
\hline Corresponding Author: & $\begin{array}{l}\text { Andrea Fabbri, MD, PhD } \\
\text { Universita degli Studi di Roma Tor Vergata Dipartimento di Medicina dei Sistemi } \\
\text { ITALY }\end{array}$ \\
\hline First Author: & Marco Infante, MD \\
\hline \multirow[t]{7}{*}{ Order of Authors: } & Marco Infante, MD \\
\hline & Barry Sears, PhD \\
\hline & Angela Maria Rizzo, PhD \\
\hline & Daniela Mariani Cerati, MD \\
\hline & Massimiliano Caprio, MD, PhD \\
\hline & Camillo Ricordi, MD \\
\hline & Andrea Fabbri, MD, PhD \\
\hline Abstract: & $\begin{array}{l}\text { Introduction: Autism spectrum disorder (ASD) is a group of neurodevelopmental } \\
\text { disorders characterized by abnormal development of cognitive, social, and } \\
\text { communicative skills. Although ASD aetiology and pathophysiology are still unclear, } \\
\text { various nutritional factors have been investigated as potential risk factors for ASD } \\
\text { development, including omega-3 polyunsaturated fatty acids (PUFAs) and vitamin D } \\
\text { deficiency. In fact, both omega-3 PUFAs and vitamin D are important for brain } \\
\text { development and function. } \\
\text { Case report: Herein, we report the case of a } 23 \text {-year-old young adult male with autism } \\
\text { who was referred to our Unit due to a } 12 \text { months history of cyclic episodes of } \\
\text { restlessness, agitation, irritability, oppositional and self-injurious behaviours. } \\
\text { Laboratory tests documented a markedly altered omega-6/omega-3 balance, along } \\
\text { with a vitamin D deficiency, as assessed by serum levels of } 25 \text {-hydroxyvitamin D. } \\
\text { Omega-3 and vitamin D co-supplementation was therefore started, with remarkable } \\
\text { improvements in ASD symptoms throughout a } 24 \text { months follow-up period. A brief } \\
\text { review of the literature for interventional studies evaluating the efficacy of omega-3 or } \\
\text { vitamin D supplementation for the treatment of ASD-related symptoms is also provided. } \\
\text { Conclusion: To our knowledge, this is the first case reporting remarkable beneficial } \\
\text { effects on ASD symptoms deriving from omega-3 and vitamin D combination therapy. } \\
\text { This case report suggests omega-3 and vitamin D co-supplementation as a potential } \\
\text { safe-effective therapeutic strategy to treat core symptoms of ASD. However, larger } \\
\text { studies are needed to evaluate the real effectiveness of such therapeutic approach in a } \\
\text { broader sample of ASD patients. }\end{array}$ \\
\hline
\end{tabular}




\title{
Omega-3 PUFAs and vitamin $D$ co-supplementation as a safe-effective therapeutic approach for core symptoms of autism spectrum disorder: case report and literature review
}

\author{
Authors: \\ Marco Infante $^{1}$, Barry Sears ${ }^{2}$, Angela Maria Rizzo ${ }^{3}$, Daniela Mariani Cerati $^{4}$, Massimiliano Caprio ${ }^{5,6}$, \\ Camillo Ricordi ${ }^{7,8}$, Andrea Fabbri ${ }^{1}$
}

\section{Affiliations:}

${ }^{1}$ Unit of Endocrinology and Metabolic Diseases, Department of Systems Medicine, CTO Andrea Alesini Hospital, ASL Roma 2, University Tor Vergata, Rome, Italy. Via San Nemesio, 21, 00145, Rome, Italy.

${ }^{2}$ Inflammation Research Foundation, Peabody, MA, USA. 200 Corporate Place, Peabody, MA 01960, USA.

${ }^{3}$ Department of Pharmacological and Biomolecular Sciences, Laboratory of Membrane Biochemistry and Applied Nutrition, Milan University, Milan, Italy. Via Trentacoste 2, 20134, Milan, Italy.

${ }^{4}$ ANGSA, Associazione Nazionale Genitori Soggetti Autistici

${ }^{5}$ Laboratory of Cardiovascular Endocrinology, IRCCS San Raffaele Pisana, Rome, Italy. Via di Val Cannuta, 247, 00166, Rome, Italy.

${ }^{6}$ Department of Human Sciences and Promotion of the Quality of Life, San Raffaele Roma Open University, Rome, Italy. Via di Val Cannuta, 247, 00166, Rome, Italy.

${ }^{7}$ Diabetes Research Institute and Cell Transplant Program, University of Miami Miller School of Medicine, Miami, FL, USA. 1450 N.W. 10th Avenue, Miami, FL 33136, USA. 
${ }^{8}$ Diabetes Research Institute Federation

\section{Corresponding Author:}

Prof. Andrea Fabbri

E-mail: andrea.fabbri@uniroma2.it

Telephone: +390651003118

Fax: +390651003118

Word count: 6488 words

\section{Funding details}

This research received no specific grant from any funding agency in the public, commercial, or notfor-profit sectors.

\section{Disclosure statement}

Disclosure of interest: Dr. Sears is the President of Zone Labs, a medical food company. The other authors declare that they have no conflict of interest to disclose. 


\title{
Omega-3 PUFAs and vitamin D co-supplementation as a safe-effective therapeutic approach for core symptoms of autism spectrum disorder: case report and literature review
}

\begin{abstract}
Introduction: Autism spectrum disorder (ASD) is a group of neurodevelopmental disorders characterized by abnormal development of cognitive, social, and communicative skills. Although ASD aetiology and pathophysiology are still unclear, various nutritional factors have been investigated as potential risk factors for ASD development, including omega-3 polyunsaturated fatty acids (PUFAs) and vitamin D deficiency. In fact, both omega-3 PUFAs and vitamin D are important for brain development and function.
\end{abstract}

Case report: Herein, we report the case of a 23 -year-old young adult male with autism who was referred to our Unit due to a 12 months history of cyclic episodes of restlessness, agitation, irritability, oppositional and self-injurious behaviours. Laboratory tests documented a markedly altered omega6/omega-3 balance, along with a vitamin D deficiency, as assessed by serum levels of 25hydroxyvitamin D. Omega-3 and vitamin D co-supplementation was therefore started, with remarkable improvements in ASD symptoms throughout a 24 months follow-up period. A brief review of the literature for interventional studies evaluating the efficacy of omega-3 or vitamin D supplementation for the treatment of ASD-related symptoms is also provided.

Conclusion: To our knowledge, this is the first case reporting remarkable beneficial effects on ASD symptoms deriving from omega-3 and vitamin D combination therapy. This case report suggests omega-3 and vitamin D co-supplementation as a potential safe-effective therapeutic strategy to treat core symptoms of ASD. However, larger studies are needed to evaluate the real effectiveness of such therapeutic approach in a broader sample of ASD patients. 


\section{Keywords}

Autism, ASD, omega-3 PUFAs, omega-6 PUFAs, AA/EPA ratio, vitamin D deficiency, omega-3 supplementation, vitamin D supplementation

\section{Introduction}

Autism Spectrum Disorder (ASD) is a group of heterogeneous neurodevelopmental disorders occurring during the first years of life and characterized by abnormal development of cognitive, social, and communicative skills, often presenting with restricted patterns of interests, repetitive behaviours or movements, and sensory processing abnormalities $(1,2)$. To date, pharmacological treatments for ASD are particularly limited and almost exclusively targeted to their symptoms (3). Current limitations in development of novel treatments are certainly represented by the hazy ASD pathophysiology, as well as the paucity of clear diagnostic biomarkers and outcome measures for clinical trials $(3,4)$. Nonetheless, the prevalence of ASD dramatically raised over the last four decades (5). Even though genetic factors play a critical role in ASD (6), the aetiology of autism is likely to be multifactorial (7). In fact, the interaction between genetic and environmental factors seems to cause most of ASD (8). During the last years, the role of different environmental factors (e.g. nutritional, medications, maternal infections during pregnancy, etc.) in ASD has been extensively reviewed, with a growing interest in nutritional factors (9). Of note, the role of omega-3 and omega-6 polyunsaturated fatty acids (PUFAs) and vitamin D in development, prevention and treatment of ASD has been largely investigated (10-13).

Omega-3 PUFAs are essential nutrients that need to be provided through dietary sources or supplements (14). Importantly, omega-3 are major components of neuronal membranes and regulate important properties, such as neurite growth, neuronal survival, membrane fluidity, neurotransmission, neurogenesis, and synaptic plasticity (15-17). Hence, omega-3 are deemed necessary for a proper brain development (18). Preclinical studies showed that omega-3 deficiency 
during development affects learning and cognitive behaviour (19, 20). Furthermore, omega-3 deficiency appears to be involved in different psychiatric disorders, including ASD (21-23). Eicosapentaenoic acid (EPA), docosahexaenoic acid (DHA) and $\alpha$-linolenic acid (ALA) are highly concentrated in the brain, where they play neuroprotective, anti-oxidant, and anti-inflammatory roles (15). In particular, EPA and DHA are the precursors of specific autacoids known as resolvins, which are involved in the resolution of inflammation (24). Conversely, omega-6 PUFAs (particularly arachidonic acid, AA) compete with omega-3 and exert opposing properties, promoting inflammation, thrombosis and vasoconstriction through their derived-metabolite eicosanoids $(25,26)$. Modern western diets have progressively evolved toward a ratio of omega- 6 to omega- 3 of approximately $15 / 1$ - 16.7/1 (25). Importantly, the increased ratio of omega- 6 to omega-3 has been shown to contribute to pathogenesis of several disease, such as cancer, obesity, cardiovascular disease, and autoimmune and inflammatory disorders $(14,25,27)$. This could have relevant implications even for ASD, which is characterized by reduced cerebral blood flow and neuroinflammation $(28,29)$. Moreover, the marked rise in the prevalence of ASD observed during last decades appears to parallel dietary changes in essential fatty acids intake, which has gradually shifted towards higher amounts of omega- 6 compared to omega-3 (30), suggesting the omega-3 deficient diet as a potential risk factor for ASD development (10). Evidence also shows that children with ASD have lower levels of plasma and erythrocyte membrane omega-3, as well as a higher AA/EPA ratio compared with healthy controls $(22,31)$. These findings could be related to low omega3 dietary intake and/or differences in fatty acid metabolism and incorporation into cell membranes of ASD children (31).

Vitamin D production is mostly UVB-mediated through adequate sun exposure, with a minor fraction secured by dietary intake (32). According to the Endocrine Society guidelines, vitamin D deficiency is defined as serum levels of $25(\mathrm{OH}) \mathrm{D}$ (25-hydroxyvitamin $\mathrm{D}$, the major circulating metabolite of vitamin D) below $50 \mathrm{nmol} / \mathrm{L}$, whereas vitamin D insufficiency is defined as serum levels ranged 
between 52.5 and $72.5 \mathrm{nmol} / \mathrm{L}$ (33). Vitamin D has been shown to play several extra-skeletal effects, other than the well-known regulation of calcium-phosphorus and bone homeostasis (34). Importantly, a growing body of evidence suggests an involvement of vitamin D deficiency in $\operatorname{ASD}(12,13)$. The autism-vitamin D theory has been first introduced by Cannell, which proposed gestational and early childhood vitamin D deficiency as the primary environmental trigger for autism development (35). Vitamin D acts as a neuroactive steroid playing an important role in brain development and mature brain function $(36,37)$. Accordingly, both vitamin D receptor (VDR) and 1 $\alpha$-hydroxylase - the enzyme responsible for the synthesis of the active form of vitamin D, namely 1,25-dihydroxyvitamin D - have been found in human brain (38). Moreover, autism prevalence is higher in areas of lower UVB penetration (39). Different studies and meta-analyses have shown that children and adults with ASD exhibit significantly lower levels of serum $25(\mathrm{OH}) \mathrm{D}$ compared to healthy controls, further suggesting that vitamin D deficiency represents a risk factor for ASD (40-43). Furthermore, lower levels of $25(\mathrm{OH}) \mathrm{D}$ have been related to higher severity of autism-related symptoms $(44,45)$. Maternal vitamin D deficiency has also been associated with increased offspring risk for ASD development $(46,47)$. Thus, the growing increase in the prevalence of vitamin D deficiency - observed not only in adults, but even in infants, children and adolescents $(48,49)$ - might correspond to the apparent increase in the prevalence of ASD during the last decades (5).

Herein we describe the case of a 23-year-old young adult male with autism, who was referred to our Unit for progressive worsening of psychiatric symptoms, which gradually and substantially improved after vitamin D and omega-3 combination therapy initiation throughout a 24 months follow-up period. Written informed consent was given by the parents for permission to publish this case report. In light of this report, we also provided a brief review of the literature for interventional studies evaluating the efficacy of omega-3 or vitamin D supplementation for the treatment of ASD-related symptoms. 


\section{Materials and Methods}

Serum 25(OH)D levels were measured by radioimmunoassay on the Gamma Counter Wizard 1470. AA/EPA ratio derived from the fatty acid composition obtained from a finger stick drop of whole blood, which has been then analysed by gas-chromatographic analysis $(50,51)$. The Childhood Autism Rating Scale (CARS) has been administered to evaluate the degree of autism-related symptoms. It consists of 14 items assessing the autism-related behaviours and a $15^{\text {th }}$ item rating general impressions of the disease. Total scores range from a minimum of 15 to a maximum of 60 , whit higher scores being associated with a higher level of impairment. In particular, scores below 30 correspond to the non-autistic range, scores between 30 and 36.5 correspond to mild to moderate autism, whereas scores $\geq 37$ indicate severe autism $(52,53)$. The Clinical Global Impressions (CGI) Scale has been administered to quantify and track patient's clinical progress and treatment response during the follow-up. It consists of two components: i) CGI-Severity (CGI-S), which assesses the average severity of symptoms basing upon observed and reported symptoms, behaviour, and function during the seven days just prior to the baseline visit, and ii) CGI-Improvement (CGI-I), which is aimed at comparing the patient's clinical condition at any time after treatment initiation to the 1-week period just prior to the baseline visit. CGI-S scores range from a low of 1 to a high of 7, with score 1 indicating a normal mental status and score 7 corresponding to the highest severity of symptoms. CGI-I scores range from a low of 1 to a high of 7, with score 1 indicating the best improvement after treatment initiation, and score 7 corresponding to the most severe clinical worsening after treatment initiation (54).

\section{Case description}

We report the case of a 23-year-old young adult male with autism, who was referred to the Unit of Endocrinology and Metabolic Diseases of the CTO Andrea Alesini Hospital (Department of Systems Medicine, University Tor Vergata, Rome, Italy) due to a 12 months history of cyclic episodes of 
restlessness, agitation, irritability, oppositional and self-injurious behaviours. These episodes lasted for approximately 6-8 consecutive days each month. The patient was born at full term, after uncomplicated pregnancy and eutocic delivery. He was diagnosed with autism at the age of 3 years and 6 months by a team of neuropsychiatrists, according to the DSM-IV diagnostic criteria (55). Family history was negative for ASD or other psychiatric disorders. At the admission, the patient had been taking melatonin ( $3 \mathrm{mg} / \mathrm{day})$ for insomnia and the second-generation antipsychotic risperidone ( $1 \mathrm{mg} /$ day) for irritability since the age of 11 years. Moreover, he was also undergoing a cognitive behavioural therapy program. First physical examination revealed normal blood pressure (120/70 $\mathrm{mmHg})$, heart rate $(80 \mathrm{bpm})$ and body mass index (BMI 22.9; weight $70 \mathrm{Kg}$, height $177 \mathrm{~cm}$ ). CARS total score at the baseline visit was 37, indicating a condition of severe autism. CGI-S score was 5, indicating a condition of markedly ill mental status. Laboratory tests showed normal results, except for low serum levels of 25(OH)D (37.75 nmol/L; nv > $75 \mathrm{nmol} / \mathrm{L})$, indicative of vitamin $\mathrm{D}$ deficiency, and the omega-6/omega-3 (AA/EPA) ratio, which was very high $(49.54, \mathrm{nv}<3.0)$. In light of these findings, the patient was started on cholecalciferol plus highly purified omega-3 PUFAs supplementation. Cholecalciferol prescribed dose was 25.000 IU weekly in form of one single-dose oral solution (Dibase®; cholecalciferol $25.000 \mathrm{IU} / 2.5 \mathrm{~mL}$ ). This dose was aimed at achieving target $25(\mathrm{OH}) \mathrm{D}$ serum levels $\geq 75 \mathrm{nmol} / \mathrm{L}$, which were recently reported as the minimum levels able to induce significant clinical improvement in ASD patients (44). Omega-3 supplementation consisted in the administration of ultra-refined omega-3 EPA + DHA concentrate (EnerZona® Omega 3 Rx; EPA $400 \mathrm{mg}$ and DHA $200 \mathrm{mg}$ per $1 \mathrm{~g}$ capsule, 2 capsules/three times daily), for a total EPA/DHA dose of $2.400 / 1.200 \mathrm{mg}$ daily, corresponding to approximately $50 \mathrm{mg}$ of EPA and DHA/ $\mathrm{kg}$ body weight per day. This dose was aimed at achieving a target AA/EPA ratio $<3$, according to previous findings observed in different clinical settings $(50,56-58)$. The patient was monitored for a total follow-up period of 24 months. Target $25(\mathrm{OH}) \mathrm{D}$ serum concentrations were progressively achieved after cholecalciferol administration, with $25(\mathrm{OH}) \mathrm{D}$ serum levels of $59.25 \mathrm{nmol} / \mathrm{L}, 70 \mathrm{nmol} / \mathrm{L}, 145.25$ $\mathrm{nmol} / \mathrm{L}, 108.25 \mathrm{nmol} / \mathrm{L}$, and $105.5 \mathrm{nmol} / \mathrm{L}$, at 3, 6, 12, 18, and 24 months post-baseline visit, 
respectively. Since the omega-3 supplementation initiation, AA/EPA gradually decreased from 49.54 at baseline to 1.96 at 24 months post-baseline visit. Table 1 shows the changes in blood fatty acids composition and serum 25(OH)D observed during the follow-up. As AA/EPA ratio and serum $25(\mathrm{OH}) \mathrm{D}$ concentrations progressively improved, remarkable improvements in symptoms were also gradually observed. In particular, restlessness, agitation, irritability, and self-injurious behaviours gradually disappeared. These improvements have been quantified through CARS total score, which dropped from a score suggestive of severe autism at the baseline (CARS total score: 38 ) to scores suggestive of mild to moderate autism at 12 and 24 months (35 and 33.5, respectively; see Figure 1). Specifically, improvements involved the following CARS items: relating to people, imitation, emotional response, body use, object use, visual response, nonverbal communication, and activity level (Figure 2). Moreover, CGI-I was administered at 3, 6, 12, 18, and 24 months, with total scores of $3,2,1,1$, and 1 , respectively (Figure 1). Risperidone dose was progressively reduced during the follow-up (0.5 mg/day at 12 months) and definitively discontinued at 18 months, according to the neuropsychiatrist prescription. Melatonin dose and cognitive behavioural therapy program were not modified during the follow-up period. At 24 months post-baseline visit, the patient was still taking oral omega-3 and cholecalciferol co-supplementation at the same doses as initially prescribed. Importantly, no side effects were reported throughout the follow-up.

\section{Discussion of the case and literature review}

Clinical benefits from omega-3 supplementation have been demonstrated in various psychiatric disorders $(23,59,60)$. Several clinical trials have been conducted to evaluate whether omega-3 can improve the core symptoms of ASD, with not univocal results (11). Four studies found improvements - although not statistically significant - in some of the ASD symptoms, such as irritability and hyperactivity/stereotypy (61-64). A small open-label pilot study has previously reported improvements in ASD symptoms after omega-3 supplementation, according to different rating scales (65). Conversely, Politi et al. found no significant clinical improvement after omega-3 
supplementation in a group of nineteen young adults with severe autism (66). It is worth to note that the limited samples size and heterogeneity in study designs may, at least in part, account for the controversial results observed in different studies (11). A recent randomized, double-blind, placebocontrolled trial conducted on children and adolescents with ASD demonstrated that omega-3 supplementation is able to improve the erythrocyte membrane omega- 6 to omega- 3 ratio, without changing plasma antioxidant status. Social motivation also improved during the study, with no significant treatment effect (67). Finally, two meta-analyses assessing the effectiveness of omega-3 fatty acids in patients with ASD showed that omega-3 supplementation leads to significant improvements in different ASD symptoms, including hyperactivity, lethargy, stereotypy, and repetitive and restricted interests and behaviours $(68,69)$.

On the other hand, data on the effectiveness of vitamin D supplementation in ASD are less univocal. The clinical evidence that vitamin D supplementation can improve core symptoms in autism was first reported by Jia et $a l$. in 2015. The authors described the case of a 32-month-old boy with ASD and severe vitamin D deficiency, whose autism-related symptoms significantly improved after cholecalciferol supplementation (150.000 IU administered intramuscularly every month, associated with 400 IU per day administered orally) during a 2-months follow-up period (70). Then, a 3-months cross-sectional study conducted on 122 ASD children found that cholecalciferol supplementation at the dose of $300 \mathrm{IU} / \mathrm{kg} /$ day (not to exceed $5.000 \mathrm{IU} /$ day) significantly improved CARS scores when target serum $25(\mathrm{OH}) \geq 75 \mathrm{nmol} / \mathrm{L}$ was achieved (44). Similar findings have been recently replicated in a double-blinded randomized clinical trial conducted on 109 ASD children, where cholecalciferol administration at a dose of $300 \mathrm{IU} / \mathrm{kg} /$ day for 4 months led to a significant improvement in autism symptoms (71). Finally, an open label study conducted on thirty-seven children with ASD and vitamin D deficiency showed that cholecalciferol administration at large bolus doses (150.000 IU/month administered intramuscularly) together with an oral dose of 400 IU/day gave rise to remarkable reductions in CARS scores, with no evidence of toxicity (45). 
Improvement in ASD symptoms deriving from omega-3 and vitamin D co-supplementation may be explained by different mechanisms basing upon the ASD pathophysiology. Indeed, autistic individuals exhibit abnormalities in immune function at both peripheral blood and brain level, with increased levels of pro-inflammatory cytokines and oxidative stress, ultimately resulting in neuroinflammation (72-74). Moreover, the presence of multiple brain-specific autoantibodies in ASD children and in their mothers suggests a potential involvement of autoimmune response in the pathogenesis of ASD (75). Therefore, omega-3 may be effective for the treatment of ASD symptoms, due to their ability to reduce neuroinflammation and oxidative stress (76-78). Similarly, vitamin D may reduce the severity of ASD symptoms targeting the neuroinflammation through its antiinflammatory properties (34), increasing regulatory $\mathrm{T}$ cells and upregulating the master antioxidant agent glutathione (79-81). Vitamin D may also exert a protective effect against the autoimmune response, up-regulating the anti-inflammatory cytokine IL-10 (82). 1,25-dihydroxyvitamin D can affect the altered neurotransmitters profile typically observed in patients with ASD, increasing brain levels of serotonin and $\gamma$-aminobutyric acid (GABA) (83-86). Vitamin D has also been shown to protect brain tissue by promoting the production of neurotrophins, which counteract toxicity from different sources (87). Finally, omega-3 and vitamin D may exert a stronger synergistic effect on oxidative stress, and inflammatory and autoimmune responses, as reported in different clinical settings $(50,57,58,88)$.

To date, there is only an ongoing trail assessing the effects of omega-3 and vitamin D cosupplementation (722 mg of DHA + 2000 IU of cholecalciferol per day) on symptoms severity in ASD children, with results being awaited (89). To our knowledge, this is the first case reporting beneficial effects of omega-3 and vitamin D combination therapy on ASD symptoms, as proved by the marked improvements in CARS and CGI-I scores observed throughout the follow-up. Notably, clinical improvement corresponded to the progressive decrease of AA/EPA ratio and increase of DHA, along with the gradual increase of serum 25(OH)D above $100 \mathrm{nmol} / \mathrm{L}$ (Table 1). These findings 
prompted us to make some considerations. As we previously mentioned, in patients with ASD AA/EPA ratio and blood levels of omega-3 PUFAs have been found to be higher and lower, respectively, compared to healthy controls $(22,31)$. Thus, AA/EPA ratio could be proposed as a diagnostic, prognostic and therapeutic biomarker in patients with ASD, representing a useful tool to titrate the dose of omega-3 fatty acids. Moreover, patients with higher AA/EPA ratio and/or lower omega-3 levels at baseline might show a larger therapeutic effect from omega-3 supplementation, as previously reported (67). It has been documented that healthy subjects taking $5 \mathrm{~g}$ of EPA and DHA per day for 10 weeks undergo an approximately $90 \%$ reduction of the AA/EPA ratio, as well as a significant decrease in the levels of pro-inflammatory cytokines (90). In this case study, we used total daily doses of EPA and DHA (50 mg of EPA and DHA/kg body weight per day) similar to those used previously in type 1 diabetes settings (55-70 mg of EPA and DHA/kg body weight per day), which successfully reduced AA/EPA ratio below $3(50,57,58)$. It is also worth to note that different studies have shown that children with attention deficit hyperactivity disorder (ADHD) can tolerate higher daily doses of omega-3 (250-325 mg of EPA and DHA/kg body weight per day), maintaining the AA/EPA ratio below $3(59,91)$. Furthermore, an ongoing trial evaluating the effectiveness of omega3 and vitamin D co-supplementation in halting autoimmunity and disease progression in patients with type 1 diabetes (POSEIDON, Pilot Study of OMEGA-3 and Vitamin D in High-Dose in Type 1 Diabetic Patients; ClinicalTrials.gov Identifier: NCT03406897) uses even greater doses of omega-3 supplementation (150 mg of EPA and DHA/kg body weight per day) (92).

In addition, similar to AA/EPA ratio, baseline serum $25(\mathrm{OH}) \mathrm{D}$ could also represent a potential predictive biomarker for vitamin D supplementation efficacy in ASD symptoms. Indeed, different studies and meta-analyses have found lower levels of serum $25(\mathrm{OH}) \mathrm{D}$ in children and adults with ASD compared to healthy controls (40-43). With regard to the therapeutic strategy, omega-3 and vitamin D combination therapy might be more effective than omega-3 or vitamin D supplementation administered alone, potentially due to their synergistic actions on oxidative stress and inflammatory 
parameters $(50,57,58,88)$. Furthermore, most of the interventional studies on omega-3 and vitamin D supplementation have been conducted in children. Nonetheless, potential beneficial effects from this therapeutic strategy may be documented even in young adult patients with ASD, as demonstrated by our case. Finally, the presence of a specific subgroup of ASD patients and/or symptom clusters may predict the responsiveness to omega-3 and vitamin D co-supplementation. Large clinical trials with a proper study duration are needed to evaluate and/or confirm all the aforementioned hypotheses.

\section{Conclusion}

To our knowledge, this is this first case reporting significant beneficial effects of omega- 3 and vitamin

D co-supplementation on autism-related symptoms in a young adult patient. Such therapeutic approach could represent a safe and effective combination therapy to treat core symptoms and improve the quality of life in patients with ASD. This case report further underlines the importance of assessment of serum $25(\mathrm{OH}) \mathrm{D}$ concentrations in ASD management, in order to early detect a vitamin D deficiency and subsequently start an adequate vitamin D supplementation. We believe that also the AA/EPA ratio could represent a valid diagnostic, prognostic and therapeutic biomarker to start a proper omega-3 supplementation and to improve ASD-related symptoms. 


\section{References}

1. Grzadzinski R, Huerta M, Lord C. DSM-5 and autism spectrum disorders (ASDs): an opportunity for identifying ASD subtypes. Mol Autism. 2013;4(1):12.

2. Volkmar F, Siegel M, Woodbury-Smith M, King B, McCracken J, State M, et al. Practice parameter for the assessment and treatment of children and adolescents with autism spectrum disorder. J Am Acad Child Adolesc Psychiatry. 2014;53(2):237-57.

3. LeClerc S, Easley D. Pharmacological therapies for autism spectrum disorder: a review. P T. 2015;40(6):389-97.

4. Persico AM, Arango C, Buitelaar JK, Correll CU, Glennon JC, Hoekstra PJ, et al. Unmet needs in paediatric psychopharmacology: Present scenario and future perspectives. Eur Neuropsychopharmacol. 2015;25(10):1513-31.

5. Zablotsky B, Black LI, Maenner MJ, Schieve LA, Blumberg SJ. Estimated Prevalence of Autism and Other Developmental Disabilities Following Questionnaire Changes in the 2014 National Health Interview Survey. Natl Health Stat Report. 2015(87):1-20.

6. Shailesh H, Gupta I, Sif S, Ouhtit A. Towards understanding the genetics of Autism. Front Biosci (Elite Ed). 2016;8:412-26.

7. Watts TJ. The pathogenesis of autism. Clin Med Pathol. 2008;1:99-103.

8. Chaste P, Leboyer M. Autism risk factors: genes, environment, and gene-environment interactions. Dialogues Clin Neurosci. 2012;14(3):281-92.

9. Modabbernia A, Velthorst E, Reichenberg A. Environmental risk factors for autism: an evidencebased review of systematic reviews and meta-analyses. Mol Autism. 2017;8:13.

10. Field SS. Interaction of genes and nutritional factors in the etiology of autism and attention deficit/hyperactivity disorders: a case control study. Med Hypotheses. 2014;82(6):654-61. 
11. Agostoni C, Nobile M, Ciappolino V, Delvecchio G, Tesei A, Turolo S, et al. The Role of Omega3 Fatty Acids in Developmental Psychopathology: A Systematic Review on Early Psychosis, Autism, and ADHD. Int J Mol Sci. 2017;18(12).

12. Cannell JJ. Vitamin D and autism, what's new? Rev Endocr Metab Disord. 2017;18(2):183-93.

13. Jia F, Shan L, Wang B, Li H, Miao C, Xu Z, et al. Bench to bedside review: Possible role of vitamin D in autism spectrum disorder. Psychiatry Res. 2018;260:360-5.

14. Simopoulos AP. The importance of the omega-6/omega-3 fatty acid ratio in cardiovascular disease and other chronic diseases. Exp Biol Med (Maywood). 2008;233(6):674-88.

15. Crupi R, Marino A, Cuzzocrea S. n-3 fatty acids: role in neurogenesis and neuroplasticity. Curr Med Chem. 2013;20(24):2953-63.

16. Parletta N, Milte CM, Meyer BJ. Nutritional modulation of cognitive function and mental health. J Nutr Biochem. 2013;24(5):725-43.

17. Dyall SC. Long-chain omega-3 fatty acids and the brain: a review of the independent and shared effects of EPA, DPA and DHA. Front Aging Neurosci. 2015;7:52.

18. Luchtman DW, Song C. Cognitive enhancement by omega-3 fatty acids from child-hood to old age: findings from animal and clinical studies. Neuropharmacology. 2013;64:550-65.

19. Moriguchi T, Greiner RS, Salem N. Behavioral deficits associated with dietary induction of decreased brain docosahexaenoic acid concentration. J Neurochem. 2000;75(6):2563-73.

20. Salem N, Litman B, Kim HY, Gawrisch K. Mechanisms of action of docosahexaenoic acid in the nervous system. Lipids. 2001;36(9):945-59.

21. McNamara RK, Perry M, Sears B. Dissociation of C-Reactive Protein Levels from Long-Chain Omega-3 Fatty Acid Status and Antidepressant Response in Adolescents with Major Depressive Disorder: An Open-Label Dose-Ranging Trial. Journal of Nutritional Therapeutics, 2013, 2, 000-000. 22. Parletta N, Niyonsenga T, Duff J. Omega-3 and Omega-6 Polyunsaturated Fatty Acid Levels and Correlations with Symptoms in Children with Attention Deficit Hyperactivity Disorder, Autistic Spectrum Disorder and Typically Developing Controls. PLoS One. 2016;11(5):e0156432. 
23. Grosso G, Galvano F, Marventano S, Malaguarnera M, Bucolo C, Drago F, et al. Omega-3 fatty acids and depression: scientific evidence and biological mechanisms. Oxid Med Cell Longev. 2014;2014:313570.

24. Spite M, Clària J, Serhan CN. Resolvins, specialized proresolving lipid mediators, and their potential roles in metabolic diseases. Cell Metab. 2014;19(1):21-36.

25. Simopoulos AP. The importance of the ratio of omega-6/omega-3 essential fatty acids. Biomed Pharmacother. 2002;56(8):365-79.

26. Serhan CN. Pro-resolving lipid mediators are leads for resolution physiology. Nature. 2014;510(7503):92-101.

27. Simopoulos AP. An Increase in the Omega-6/Omega-3 Fatty Acid Ratio Increases the Risk for Obesity. Nutrients. 2016;8(3):128.

28. Yang WH, Jing J, Xiu LJ, Cheng MH, Wang X, Bao P, et al. Regional cerebral blood flow in children with autism spectrum disorders: a quantitative ${ }^{9}{ }^{9}$ mTc-ECD brain SPECT study with statistical parametric mapping evaluation. Chin Med J (Engl). 2011;124(9):1362-6.

29. Dipasquale V, Cutrupi MC, Colavita L, Manti S, Cuppari C, Salpietro C. Neuroinflammation in Autism Spectrum Disorders: Role of High Mobility Group Box 1 Protein. Int J Mol Cell Med. 2017;6(3):148-55.

30. van Elst K, Bruining H, Birtoli B, Terreaux C, Buitelaar JK, Kas MJ. Food for thought: dietary changes in essential fatty acid ratios and the increase in autism spectrum disorders. Neurosci Biobehav Rev. 2014;45:369-78.

31. Vancassel S, Durand G, Barthélémy C, Lejeune B, Martineau J, Guilloteau D, et al. Plasma fatty acid levels in autistic children. Prostaglandins Leukot Essent Fatty Acids. 2001;65(1):1-7.

32. Webb AR, Pilbeam C, Hanafin N, Holick MF. An evaluation of the relative contributions of exposure to sunlight and of diet to the circulating concentrations of 25-hydroxyvitamin D in an elderly nursing home population in Boston. Am J Clin Nutr. 1990;51(6):1075-81. 
33. Holick MF, Binkley NC, Bischoff-Ferrari HA, Gordon CM, Hanley DA, Heaney RP, et al. Evaluation, treatment, and prevention of vitamin D deficiency: an Endocrine Society clinical practice guideline. J Clin Endocrinol Metab. 2011;96(7):1911-30.

34. Caprio M, Infante M, Calanchini M, Mammi C, Fabbri A. Vitamin D: not just the bone. Evidence for beneficial pleiotropic extraskeletal effects. Eat Weight Disord. 2017;22(1):27-41.

35. Cannell JJ. Autism and vitamin D. Med Hypotheses. 2008;70(4):750-9.

36. Kesby JP, Eyles DW, Burne TH, McGrath JJ. The effects of vitamin D on brain development and adult brain function. Mol Cell Endocrinol. 2011;347(1-2):121-7.

37. Eyles DW, Burne TH, McGrath JJ. Vitamin D, effects on brain development, adult brain function and the links between low levels of vitamin D and neuropsychiatric disease. Front Neuroendocrinol. 2013;34(1):47-64.

38. Eyles DW, Smith S, Kinobe R, Hewison M, McGrath JJ. Distribution of the vitamin D receptor and 1 alpha-hydroxylase in human brain. J Chem Neuroanat. 2005;29(1):21-30.

39. Grant WB, Cannell JJ. Autism prevalence in the United States with respect to solar UV-B doses: An ecological study. Dermatoendocrinol. 2013;5(1):159-64.

40. Gong ZL, Luo CM, Wang L, Shen L, Wei F, Tong RJ, et al. Serum 25-hydroxyvitamin D levels in Chinese children with autism spectrum disorders. Neuroreport. 2014;25(1):23-7.

41. Fernell E, Bejerot S, Westerlund J, Miniscalco C, Simila H, Eyles D, et al. Autism spectrum disorder and low vitamin D at birth: a sibling control study. Mol Autism. 2015;6:3.

42. Endres D, Dersch R, Stich O, Buchwald A, Perlov E, Feige B, et al. Vitamin D Deficiency in Adult Patients with Schizophreniform and Autism Spectrum Syndromes: A One-Year Cohort Study at a German Tertiary Care Hospital. Front Psychiatry. 2016;7:168.

43. Wang T, Shan L, Du L, Feng J, Xu Z, Staal WG, et al. Serum concentration of 25-hydroxyvitamin D in autism spectrum disorder: a systematic review and meta-analysis. Eur Child Adolesc Psychiatry. 2016;25(4):341-50. 
44. Saad K, Abdel-Rahman AA, Elserogy YM, Al-Atram AA, Cannell JJ, Bjørklund G, et al. Vitamin D status in autism spectrum disorders and the efficacy of vitamin D supplementation in autistic children. Nutr Neurosci. 2016;19(8):346-51.

45. Feng J, Shan L, Du L, Wang B, Li H, Wang W, et al. Clinical improvement following vitamin D3 supplementation in Autism Spectrum Disorder. Nutr Neurosci. 2017;20(5):284-90.

46. Grant WB, Soles CM. Epidemiologic evidence supporting the role of maternal vitamin D deficiency as a risk factor for the development of infantile autism. Dermatoendocrinol. 2009;1(4):223-8.

47. Magnusson C, Lundberg M, Lee BK, Rai D, Karlsson H, Gardner R, et al. Maternal vitamin D deficiency and the risk of autism spectrum disorders: population-based study. BJPsych Open. 2016;2(2):170-2.

48. Huh SY, Gordon CM. Vitamin D deficiency in children and adolescents: epidemiology, impact and treatment. Rev Endocr Metab Disord. 2008;9(2):161-70.

49. Shin YH, Shin HJ, Lee YJ. Vitamin D status and childhood health. Korean J Pediatr. 2013;56(10):417-23.

50. Cadario F, Savastio S, Rizzo AM, Carrera D, Bona G, Ricordi C. Can Type 1 diabetes progression be halted? Possible role of high dose vitamin D and omega 3 fatty acids. Eur Rev Med Pharmacol Sci. 2017;21(7):1604-9.

51. Rizzo AM, Montorfano G, Negroni M, Adorni L, Berselli P, Corsetto P, et al. A rapid method for determining arachidonic:eicosapentaenoic acid ratios in whole blood lipids: correlation with erythrocyte membrane ratios and validation in a large Italian population of various ages and pathologies. Lipids Health Dis. 2010;9:7.

52. Schopler E, Reichler RJ, DeVellis RF, Daly K. Toward objective classification of childhood autism: Childhood Autism Rating Scale (CARS). J Autism Dev Disord. 1980;10(1):91-103.

53. Chlebowski C, Green JA, Barton ML, Fein D. Using the childhood autism rating scale to diagnose autism spectrum disorders. J Autism Dev Disord. 2010;40(7):787-99. 
54. Busner J, Targum SD. The clinical global impressions scale: applying a research tool in clinical practice. Psychiatry (Edgmont). 2007;4(7):28-37.

55. American Psychiatric Association. Diagnostic and Statistical Manual of Mental Disorders. 4th ed. Washington, DC: American Psychiatric Association; 1994

56. Mozaffarian D. JELIS, fish oil, and cardiac events. Lancet. 2007;369(9567):1062-3.

57. Baidal DA, Ricordi C, Garcia-Contreras M, Sonnino A, Fabbri A. Combination high-dose omega3 fatty acids and high-dose cholecalciferol in new onset type 1 diabetes: a potential role in preservation of beta-cell mass. Eur Rev Med Pharmacol Sci. 2016;20(15):3313-8.

58. Cadario F, Savastio S, Ricotti R, Rizzo AM, Carrera D, Maiuri L, et al. Administration of vitamin D and high dose of omega 3 to sustain remission of type 1 diabetes. Eur Rev Med Pharmacol Sci. 2018;22(2):512-5.

59. Sorgi PJ, Hallowell EM, Hutchins HL, Sears B. Effects of an open-label pilot study with highdose EPA/DHA concentrates on plasma phospholipids and behavior in children with attention deficit hyperactivity disorder. Nutr J. 2007;6:16.

60. Osher Y, Belmaker RH. Omega-3 fatty acids in depression: a review of three studies. CNS Neurosci Ther. 2009;15(2):128-33.

61. Amminger GP, Berger GE, Schäfer MR, Klier C, Friedrich MH, Feucht M. Omega-3 fatty acids supplementation in children with autism: a double-blind randomized, placebo-controlled pilot study. Biol Psychiatry. 2007;61(4):551-3.

62. Bent S, Bertoglio K, Ashwood P, Bostrom A, Hendren RL. A pilot randomized controlled trial of omega-3 fatty acids for autism spectrum disorder. J Autism Dev Disord. 2011;41(5):545-54.

63. Bent S, Hendren RL, Zandi T, Law K, Choi JE, Widjaja F, et al. Internet-based, randomized, controlled trial of omega-3 fatty acids for hyperactivity in autism. J Am Acad Child Adolesc Psychiatry. 2014;53(6):658-66. 
64. Mankad D, Dupuis A, Smile S, Roberts W, Brian J, Lui T, et al. A randomized, placebo controlled trial of omega-3 fatty acids in the treatment of young children with autism. Mol Autism. 2015;6:18. 65. Meiri G, Bichovsky Y, Belmaker RH. Omega 3 fatty acid treatment in autism. J Child Adolesc Psychopharmacol. 2009;19(4):449-51.

66. Politi P, Cena H, Comelli M, Marrone G, Allegri C, Emanuele E, et al. Behavioral effects of omega-3 fatty acid supplementation in young adults with severe autism: an open label study. Arch Med Res. 2008;39(7):682-5.

67. Parellada M, Llorente C, Calvo R, Gutierrez S, Lázaro L, Graell M, et al. Randomized trial of omega-3 for autism spectrum disorders: Effect on cell membrane composition and behavior. Eur Neuropsychopharmacol. 2017;27(12):1319-30.

68. Cheng YS, Tseng PT, Chen YW, Stubbs B, Yang WC, Chen TY, et al. Supplementation of omega 3 fatty acids may improve hyperactivity, lethargy, and stereotypy in children with autism spectrum disorders: a meta-analysis of randomized controlled trials. Neuropsychiatr Dis Treat. 2017;13:253143.

69. Mazahery H, Stonehouse W, Delshad M, Kruger MC, Conlon CA, Beck KL, et al. Relationship between Long Chain n-3 Polyunsaturated Fatty Acids and Autism Spectrum Disorder: Systematic Review and Meta-Analysis of Case-Control and Randomised Controlled Trials. Nutrients. 2017;9(2). 70. Jia F, Wang B, Shan L, Xu Z, Staal WG, Du L. Core symptoms of autism improved after vitamin D supplementation. Pediatrics. 2015;135(1):e196-8.

71. Saad K, Abdel-Rahman AA, Elserogy YM, Al-Atram AA, El-Houfey AA, Othman HA, et al. Randomized controlled trial of vitamin D supplementation in children with autism spectrum disorder. J Child Psychol Psychiatry. 2018;59(1):20-9.

72. James SJ, Cutler P, Melnyk S, Jernigan S, Janak L, Gaylor DW, et al. Metabolic biomarkers of increased oxidative stress and impaired methylation capacity in children with autism. Am J Clin Nutr. 2004;80(6):1611-7. 
73. Smaga I, Niedzielska E, Gawlik M, Moniczewski A, Krzek J, Przegaliński E, et al. Oxidative stress as an etiological factor and a potential treatment target of psychiatric disorders. Part 2. Depression, anxiety, schizophrenia and autism. Pharmacol Rep. 2015;67(3):569-80.

74. Szachta P, Skonieczna-Żydecka K, Adler G, Karakua-Juchnowicz H, Madlani H, Ignyś I. Immune related factors in pathogenesis of autism spectrum disorders. Eur Rev Med Pharmacol Sci. 2016;20(14):3060-72.

75. Mazur-Kolecka B, Cohen IL, Gonzalez M, Jenkins EC, Kaczmarski W, Brown WT, et al. Autoantibodies against neuronal progenitors in sera from children with autism. Brain Dev. 2014;36(4):322-9.

76. Hopperton KE, Trépanier MO, Giuliano V, Bazinet RP. Brain omega-3 polyunsaturated fatty acids modulate microglia cell number and morphology in response to intracerebroventricular amyloid- $\beta$ 1-40 in mice. J Neuroinflammation. 2016;13(1):257.

77. Inoue T, Tanaka M, Masuda S, Ohue-Kitano R, Yamakage H, Muranaka K, et al. Omega-3 polyunsaturated fatty acids suppress the inflammatory responses of lipopolysaccharide-stimulated mouse microglia by activating SIRT1 pathways. Biochim Biophys Acta Mol Cell Biol Lipids. 2017;1862(5):552-60.

78. Chen X, Wu S, Chen C, Xie B, Fang Z, Hu W, et al. Omega-3 polyunsaturated fatty acid supplementation attenuates microglial-induced inflammation by inhibiting the HMGB1/TLR4/NFКB pathway following experimental traumatic brain injury. J Neuroinflammation. 2017;14(1):143.

79. Cannell JJ. Autism, will vitamin D treat core symptoms? Med Hypotheses. 2013;81(2):195-8. 80. Huang YN, Ho YJ, Lai CC, Chiu CT, Wang JY. 1,25-Dihydroxyvitamin D3 attenuates endotoxininduced production of inflammatory mediators by inhibiting MAPK activation in primary cortical neuron-glia cultures. J Neuroinflammation. 2015;12:147.

81. Zhang Q, Cheng Y, He M, Li T, Ma Z, Cheng H. Effect of various doses of vitamin D supplementation on pregnant women with gestational diabetes mellitus: A randomized controlled trial. Exp Ther Med. 2016;12(3):1889-95. 
82. Bakdash G, van Capel TM, Mason LM, Kapsenberg ML, de Jong EC. Vitamin D3 metabolite calcidiol primes human dendritic cells to promote the development of immunomodulatory IL-10producing T cells. Vaccine. 2014;32(47):6294-302.

83. Kaneko I, Sabir MS, Dussik CM, Whitfield GK, Karrys A, Hsieh JC, et al. 1,25Dihydroxyvitamin D regulates expression of the tryptophan hydroxylase 2 and leptin genes: implication for behavioral influences of vitamin D. FASEB J. 2015;29(9):4023-35.

84. Jiang P, Zhang LH, Cai HL, Li HD, Liu YP, Tang MM, et al. Neurochemical effects of chronic administration of calcitriol in rats. Nutrients. 2014;6(12):6048-59.

85. Chakraborti B, Verma D, Karmakar A, Jaiswal P, Sanyal A, Paul D, et al. Genetic variants of MAOB affect serotonin level and specific behavioral attributes to increase autism spectrum disorder (ASD) susceptibility in males. Prog Neuropsychopharmacol Biol Psychiatry. 2016;71:123-36.

86. Robertson CE, Ratai EM, Kanwisher N. Reduced GABAergic Action in the Autistic Brain. Curr Biol. 2016;26(1):80-5.

87. Wrzosek M, Łukaszkiewicz J, Jakubczyk A, Matsumoto H, Piątkiewicz P, Radziwoń-Zaleska M, et al. Vitamin D and the central nervous system. Pharmacol Rep. 2013;65(2):271-8.

88. Razavi M, Jamilian M, Samimi M, Afshar Ebrahimi F, Taghizadeh M, Bekhradi R, et al. The effects of vitamin D and omega-3 fatty acids co-supplementation on biomarkers of inflammation, oxidative stress and pregnancy outcomes in patients with gestational diabetes. Nutr Metab (Lond). $2017 ; 14: 80$.

89. Mazahery H, Conlon C, Beck KL, Kruger MC, Stonehouse W, Camargo CA, et al. Vitamin D and omega-3 fatty acid supplements in children with autism spectrum disorder: a study protocol for a factorial randomised, double-blind, placebo-controlled trial. Trials. 2016;17(1):295.

90. Endres S, Ghorbani R, Kelley VE, Georgilis K, Lonnemann G, van der Meer JW, et al. The effect of dietary supplementation with n-3 polyunsaturated fatty acids on the synthesis of interleukin-1 and tumor necrosis factor by mononuclear cells. N Engl J Med. 1989;320(5):265-71. 
91. Germano M, Meleleo D, Montorfano G, Adorni L, Negroni M, Berra B, et al. Plasma, red blood cells phospholipids and clinical evaluation after long chain omega-3 supplementation in children with attention deficit hyperactivity disorder (ADHD). Nutr Neurosci. 2007;10(1-2):1-9.

92. Baidal DA, Sanchez J, Alejandro R, Blaschke CE, Hirani K, Matheson DL, et al. POSEIDON study: a pilot, safety and feasibility trial of high-dose omega 3 fatty acids and high-dose cholecalciferol supplementation in type 1 diabetes. CellR4 2018; 6 (1): e2489. 


\section{Tables}

Table 1 - Changes in blood fatty acid composition and serum 25(OH)D observed during the whole follow-up period (24 months). The mean values of AA, EPA and DHA (obtained from an Italian population) are shown in brackets. Abbreviations: 25(OH)D, 25-hydroxyvitamin D; AA, arachidonic acid; DHA, docosahexaenoic acid; EPA, eicosapentaenoic acid.

\begin{tabular}{|c|c|c|c|c|c|c|}
\hline & Baseline & 3 months & 6 months & 12 months & 18 months & 24 months \\
\hline $\begin{array}{l}\mathbf{A A} \\
(11.02 \pm 2.10 \%)\end{array}$ & 12.46 & 9.88 & 12.49 & 5.74 & 10.34 & 9.20 \\
\hline $\begin{array}{l}\text { EPA } \\
(0.98 \pm 0.40 \%)\end{array}$ & 0.25 & 1.96 & 3.48 & 4.30 & 3.87 & 4.70 \\
\hline $\begin{array}{l}\text { DHA } \\
(3.06 \pm 1.13 \%)\end{array}$ & 2.40 & 3.67 & 4.97 & 4.54 & 4.71 & 4.49 \\
\hline AA/EPA ratio & 49.54 & 5.05 & 3.59 & 1.34 & 2.67 & 1.96 \\
\hline $\begin{array}{l}\text { 25(OH)D } \\
\text { (deficiency: < } 50 \mathrm{nmol} / \mathrm{L}, \\
\text { insufficiency: } 52.5-72.5 \\
\text { nmol/L) }\end{array}$ & 37.75 & 59.25 & 70 & 145.25 & 108.25 & 105.5 \\
\hline
\end{tabular}




\section{Figure captions}

Figure 1 - Figure shows the patient's clinical improvement after omega-3 and vitamin D combination therapy initiation, as assessed by Childhood Autism Rating Scale (CARS) and Clinical Global Impressions (CGI) scale. CARS total score (as shown in the black box at the top right) decreased from 38 at the baseline (severe autism) to 35 and 33.5 (mild to moderate autism) at 12 and 24 months, respectively. On the other hand, Clinical Global Impressions (CGI) Scale was administered at 3, 6, 12,18 , and 24 months, with total scores of $3,2,1,1$, and 1 , respectively. It is important to note that the first score refers to CGI-Severity (CGI-S), whereas the other scores refer to CGI-Improvement (CGI-I).

Figure 2 - Schematic showing the scores of the 15 different CARS items at baseline, 12 and 24 months. Clinical improvement involved the following CARS items: relating to people, imitation, emotional response, body use, object use, visual response, nonverbal communication, and activity level. 


\section{Clinical Global Impressions (CGI) Scale}

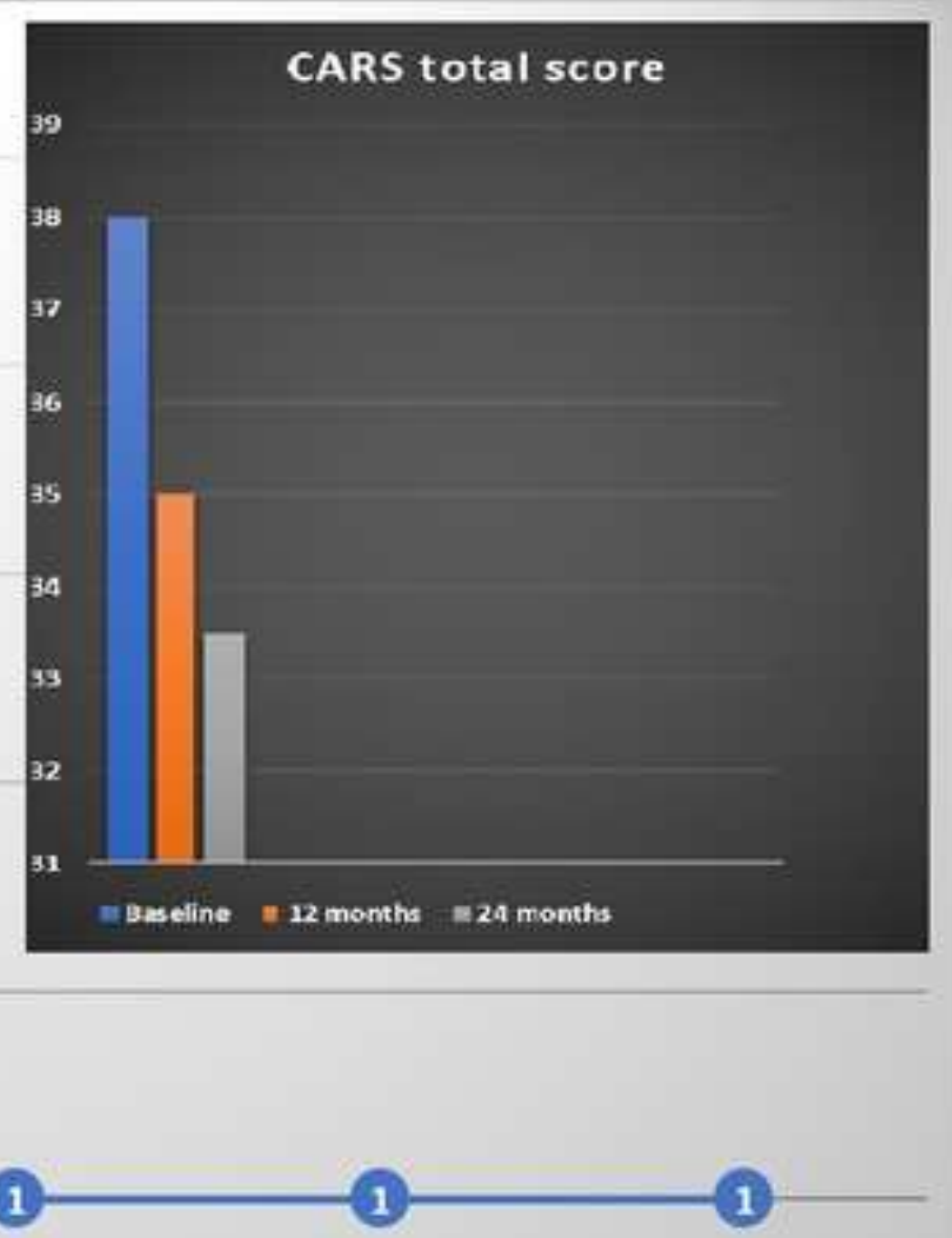




\section{Childhood Autism Rating Scale (CARS)}

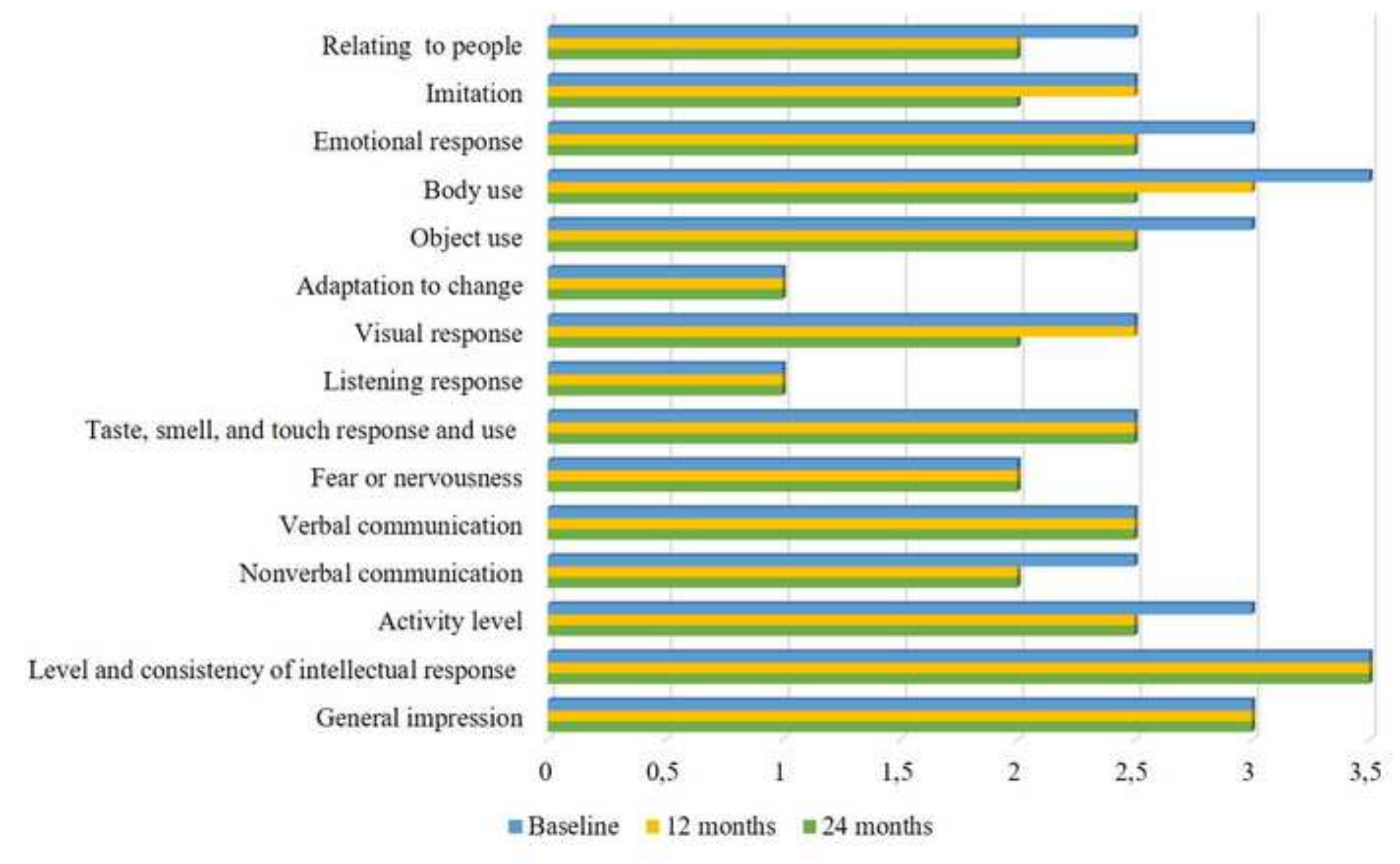

Taste, smell, and touch response and use

\section{Fear or nervousness Verbal communication Nonverbal communication Activity level}

Level and consistency of intellectual response

General impression

$=$ Baseline $=12$ months $=24$ months

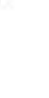

.

